\title{
Resonance production in Pomeron-Pomeron collisions at the LHC
}

\author{
R. Fiore ${ }^{1, a}$, L. Jenkovszky ${ }^{2, b}$, R. Schicker ${ }^{3, c}$ \\ ${ }_{1}^{1}$ Department of Physics, National Institute of Nuclear Physics, University of Calabria, Arcavacata di Rende, 87036 Cosenza, Italy \\ ${ }^{2}$ Bogolyubov Institute for Theoretical Physics (BITP), Ukrainian National Academy of Sciences 14-b, Metrologicheskaya str., Kiev 03680, Ukraine \\ ${ }^{3}$ Physikalisches Institut, Im Neuenheimer Feld 226, Heidelberg University, 69120 Heidelberg, Germany
}

Received: 24 November 2015 / Accepted: 11 December 2015 / Published online: 22 January 2016

(c) The Author(s) 2016. This article is published with open access at Springerlink.com

\begin{abstract}
A Regge pole model for Pomeron-Pomeron total cross section in the resonance region $\sqrt{M^{2}} \leq 5 \mathrm{GeV}$ is presented. The cross section is saturated by direct-channel contributions from the Pomeron as well as from two different $f$ trajectories, accompanied by the isolated $f_{0}(500)$ resonance dominating the $\sqrt{M^{2}} \leq 1 \mathrm{GeV}$ region. A slowly varying background is taken into account. The calculated PomeronPomeron total cross section cannot be measured directly, but is an essential part of central diffractive processes. In preparation of future calculations of central resonance production at the hadron level, and corresponding measurements at the LHC, we normalize the Pomeron-Pomeron cross section at large masses $\sigma_{t}^{P P}\left(\sqrt{M^{2}} \rightarrow \infty\right) \approx 1 \mathrm{mb}$ as suggested by QCD-motivated estimates.
\end{abstract}

\section{Introduction}

Central production in proton-proton collisions has been studied from the low energy range $\sqrt{s}=12.7-63 \mathrm{GeV}$ at the ISR at CERN up to the presently highest energy of $\sqrt{s}=13 \mathrm{TeV}$ available in Run II at the LHC. Ongoing data analysis of central production events include data taken by the COMPASS Collaboration at the SPS [1], the CDF Collaboration at the TEVATRON [2], the STAR Collaboration at RHIC [3], and the ALICE and LHCb Collaborations at the LHC $[4,5]$. A comprehensive survey of central exclusive production is given in a recent review article [6].

The analysis of central production necessitates the simulation of such events to study the acceptance and efficiency of the complex large detector systems. With the existing detector upgrade programmes for central production measurements at RHIC and at the LHC, much larger data samples are expected in the next few years which will allow for the

\footnotetext{
a e-mail: fiore@cs.infn.it

be-mail: jenk@bitp.kiev.ua

c e-mail: schicker@physi.uni-heidelberg.de
}

analysis of differential distributions. The purpose of the study presented here is the development of a Regge pole model for simulating such differential distributions.

The study of central production, in particular at the soft scale, is interesting for a variety of reasons. Here, we refer to central production as arising from the fusion of two stronlgy interacting colour-singlet objects, and we do not discuss any contributions due to photon exchange. The absence of a hard scale precludes a perturbative QCD description. The traditional framework for studying soft hadronic processes has been the Regge formalism. In this formalism, bound states are associated to Regge trajectories. The classification of mesons by means of nonlinear Regge trajectories has spectroscopic value by its own. At high energies, the hadronic interaction is dominated by the exchange of a leading trajectory, the Pomeron. Within QCD, it is conjectured that this trajectory represents the exchange of purely gluonic objects. The study of central production at high energies allows one to identify the contribution from the Pomeron trajectory. The dynamics of the corresponding multi-gluon colour-singlet exchange is presently only poorly understood within QCD, and such studies will hence contribute to an improved QCDbased understanding of Regge phenomenology. The fusion of multi-gluon objects is characterized by a gluon-dominated environment with highly suppressed quark degrees of freedom, and the evolution of this initial state is expected to populate with increased probability gluon-rich hadronic states, glueballs, and hybrids. The analysis of these centrally produced resonances by a Partial Wave Analysis reveals the quantum numbers $J^{P C}$ of these resonances. Of particular interest is the search for states with exotic quantum numbers which cannot be $q \bar{q}$-mesons, and hence must be exotic such as of tetra-quark nature $(q \bar{q}+\bar{q} q)$, or gluonic hybrid $(q \bar{q}+$ gluon $)$. Moreover, the decomposition into states of known quantum numbers will shed new light also on the existence of numerous states in the scalar sector, a topic of fundamental interest in hadron spectroscopy [7]. 
This article is organized as follows. In the introduction in Sect. 1, the study of central production at hadron colliders is motivated. In Sect. 2, central production is reviewed. In Sect. 3, the dual resonance model of Pomeron-Pomeron scattering is analysed. Nonlinear complex meson trajectories are introduced in Sect. 4. Two $f$ trajectories, relevant for the calculation of the Pomeron-Pomeron cross section, are discussed in Sect. 5, while in Sect. 6 the Pomeron trajectory is presented. In Sect. 7, the $f_{0}(500)$ resonance is examined. The Pomeron-Pomeron total cross section is investigated in Sect. 8. A summary and an outlook for more detailed studies of the topic presented here is given in Sect. 9. The procedure for fitting nonlinear complex meson trajectories is illustrated in Appendix A for the example of the $\rho$-a trajectory.

\section{Central production}

Central production in proton-proton collisions is characterized by the two forward scattered protons, or remnants thereof, and by secondary particles produced at or close to mid-rapidity. No particles are produced in the range between the mid-rapidity system and the two beam rapidities on either side of the central system. Experimentally, these event topologies can be recognized by identifying the presence of the two rapidity gaps, by detecting the forward proton or its remnants, or by a combination of these two approaches. Forward scattered neutral fragments can, for example, be detected in Zero Degree Calorimeters.

In Fig. 1, the differential cross section $\mathrm{d} \sigma / \mathrm{dP}_{t} \mathrm{dM}$ is shown for exclusive pion-pair production in the CDF Run II at the TEVATRON. Clearly seen in this picture is the resonance structure associated to the $f_{2}(1270)$. The complete kinematical determination of the final state of centrally produced pion or kaon pairs requires the measurement of the 3-momentum $\left(p_{x}, p_{y}, p_{z}\right)$ of both the positive and the negative partner of the pair. The experimental single track acceptance is, how-

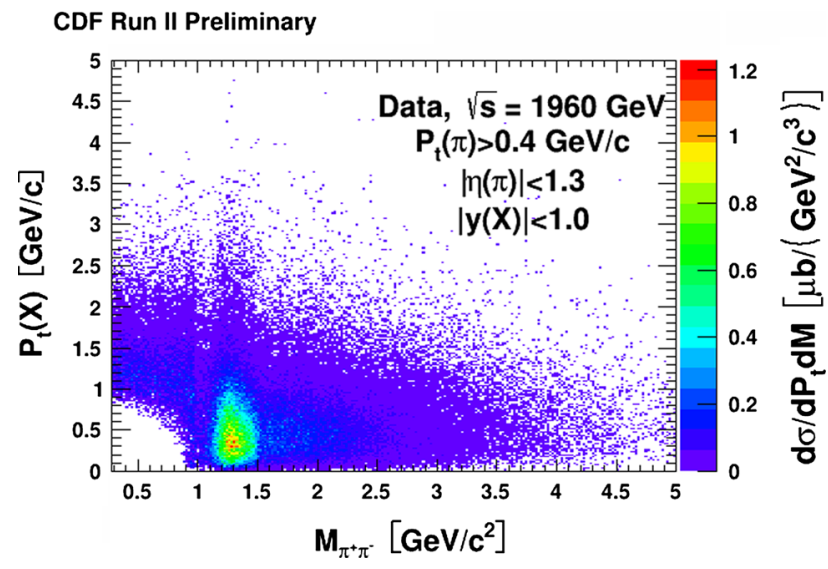

Fig. 1 Double differential cross section for central production of pion pairs measured by the CDF Collaboration (figure taken from Ref. [8]) ever, limited by finite detector coverage in pseudorapidity, as well as by a cut-off in minimum transverse momentum $\mathrm{p}_{T}$. This single track acceptance translates into missing acceptance for pairs of low mass and low transverse momentum. This missing pair acceptance is visible in Fig. 1 in the data analyzed by the CDF Collaboration. For pairs of low masses, only the high end tail of the transverse momentum distribution can be measured. The extrapolation of the cross section to low transverse momenta is, however, possible based on models which are able to reproduce the resonance structures in the part of phase space covered by the detector acceptance.

The distributions of pion-pair invariant masses measured in proton-proton collisions by the COMPASS, the CDF and the ALICE Collaboration are shown in Fig. 2 on the left, in the middle and on the right, respectively. In all these measurements, clear resonance structures are seen. At the energy of the COMPASS measurement $\sqrt{s}=18.9 \mathrm{GeV}$, Reggeons still contribute significantly to central production as evidenced in the prominent $\rho$-peak. In addition, peaks associated to the $f_{0}(980)$ and the $f_{2}(1270)$ are seen, with a broad continuum extending to the two-pion threshold. At the higher TEVATRON energy of $\sqrt{s}=1.96 \mathrm{TeV}$, the distribution at pair masses $M<900 \mathrm{MeV} / \mathrm{c}^{2}$ is significantly affected by the $\mathrm{p}_{T}$ dependence of the acceptance as shown in Fig. 1. Full acceptance down to $\mathrm{p}_{T}=0$ is reached for masses $M>900 \mathrm{MeV} / \mathrm{c}^{2}$. A clear resonance structure consistent with the $f_{2}(1270)$ is seen at around $1270 \mathrm{MeV} / \mathrm{c}^{2}$. A similar distribution is measured by the ALICE Collaboration at the LHC energy $\sqrt{s}=7 \mathrm{TeV}$ as shown in Fig. 2 on the right. In addition, the ALICE double gap measurement is compared to the pion-pair invariant mass distribution from no-gap events, i.e. from inclusive production. In inclusive production, the $\rho$ as well as the $\mathrm{K}_{s}^{0}$-signal are seen. These two signals are absent in the double-gap events, corroborating Pomeron-Pomeron dominance at the LHC energies discussed below.

The double-gap topology of central production and the relevant kinematics are shown in Fig. 3. This figure shows central production when the incoming protons remain in the ground state on the left, when one of the protons gets diffractively excited in the middle, and when both protons get excited on the right. All these reactions proceed by the exchange of Regge trajectories $\alpha\left(t_{1}\right)$ and $\alpha\left(t_{2}\right)$, which collide in the central region to produce a system of mass $M_{x}$. The total energy $s$ of the reaction is shared by the subenergies $s_{1}$ and $s_{2}$ associated to the trajectories $\alpha\left(t_{1}\right)$ and $\alpha\left(t_{2}\right)$, respectively. The LHC energies of $\sqrt{s}=7,8$ and $13 \mathrm{TeV}$ are sufficient to provide Pomeron dominance and allow for the neglect of Reggeon exchange which was not the case at the energies of previous accelerators.

The scope of the present study is the central part of the diagrams shown in Fig. 3, i.e. Pomeron-Pomeron scattering producing mesonic states of mass $M_{x}$. We isolate the PomeronPomeron-meson vertex shown in Fig. 4, and we calculate 

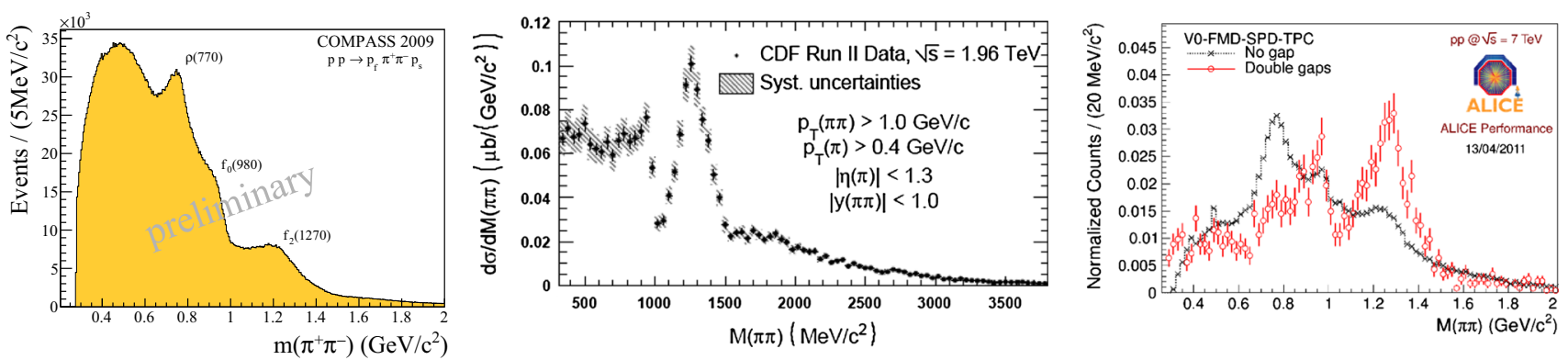

Fig. 2 Invariant pion-pair masses from the COMPASS Collaboration on the left [9], the CDF Collaboration in the middle [10], and the ALICE Collaboration on the right [11]
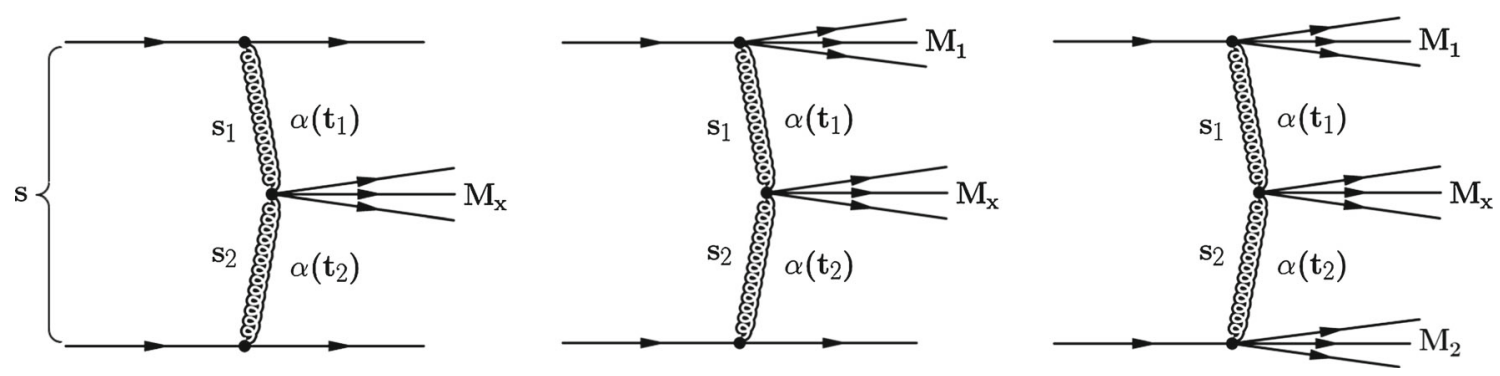

Fig. 3 Central production event topologies

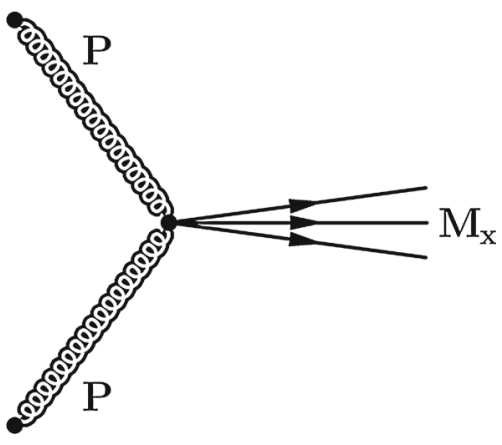

Fig. 4 Pomeron-Pomeron scattering

the Pomeron-Pomeron total cross section as a function of the centrally produced system of mass $M_{x}$. The emphasis in this study is the behaviour in the low mass resonance region where perturbative QCD approaches are not applicable. Instead, similar to $[12,13]$, we use the pole decomposition of a dual amplitude with relevant direct-channel trajectories $\alpha\left(M^{2}\right)$ for fixed values of Pomeron virtualities, $t_{1}=t_{2}=$ const. Due to Regge factorisation, the calculated Pomeron-Pomeron cross section will enter the measurable proton-proton cross section [14].

The nature of the Pomeron exchange is of fundamental interest for QCD-based studies of exchange amplitudes. An effective vectorial-exchange is very successful in reproducing the energy dependence of hadron-hadron cross sections [15]. Such an approach results in opposite signs for proton-proton and proton-antiproton amplitudes. Pomeron exchange, however, must yield the same sign for these two reaction channels. Recent studies on soft high-energy scattering solve this problem in terms of effective propagators and vertices for the Pomeron exchange [16]. Within this model, the Pomeron exchange is decribed as an effective rank-two tensor exchange $[17,18]$.

\section{Dual resonance model of Pomeron-Pomeron scattering}

The study of Pomeron-Pomeron $(P P)$ scattering is related to photon-photon scattering, the main difference being the positive and negative C-parity of the Pomeron and photon, respectively. High-virtuality $\gamma^{*} \gamma^{*}$ scattering is a favourite process in the framework of perturbative QCD, where the total cross section was calculated in Ref. [19]. In the leadingorder BFKL

$\sigma_{\text {tot }}^{\gamma^{*} \gamma^{*}}=\frac{\sigma_{0}}{\sqrt{Q_{1}^{2} Q_{2}^{2} Y}}\left(s / s_{0}\right)^{\lambda}$,

where $Q_{i}^{2}=-q_{i}^{2}, i=1,2$ is the photon virtuality and $Y=$ $\ln \left(\frac{s}{s_{0}}\right)$. The quantity $\sigma_{0}$ is a free parameter and the exponent $\lambda \equiv \alpha_{P}^{\mathrm{BFKL}}$ is the familiar BFKL eigenvalue $N_{c} \alpha_{s} 4 \ln 2 / \pi$. We recall that the transition from photon-photon to central Pomeron-Pomeron scattering is accompanied by the change of variables $Q^{2} \rightarrow t$ and $s \rightarrow M^{2}$. The above result was improved in Refs. [20,21].

Most of the studies on diffraction dissociation, single, double and central, use the triple Reggeon formalism. This approach is useful in the smooth Regge region, beyond the 


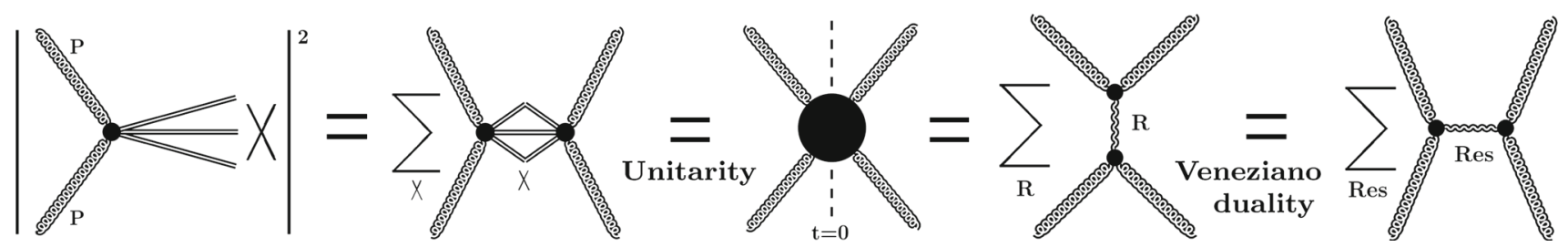

Fig. 5 Connection, through unitarity (generalized optical theorem) and Veneziano-duality, between the Pomeron-Pomeron cross section and the sum of direct-channel resonances

resonance region, but is not applicable for the production of low masses which is dominated by resonances. We solve this problem by using a dual model.

The one-by-one account of single resonances is possible, but not economic for the calculation of cross section, to which a sequence of resonances contributes at low masses. These resonances overlap and gradually disappear in the continuum at higher masses. An approach to account for many resonances, based on the idea of duality with a limited number of resonances lying on nonlinear Regge trajectories, was suggested in Ref. [22]. Later on, this approach was used in Refs. $[12,13]$ to calculate low mass single- and double-diffractive dissociation at the LHC.

The main idea behind this approach is illustrated in Fig. 5, realized by dual amplitudes with Mandelstam analyticity (DAMA) [23]. For $s \rightarrow \infty$ and fixed $t$ it is Reggebehaved. Contrary to the Veneziano model, DAMA not only allows for, but it rather requires the use of nonlinear complex trajectories providing the resonance widths via the imaginary part of the trajectory. In the case of limited real part, a finite number of resonances is produced. More specifically, the asymptotic rise of the trajectories in DAMA is limited by the condition, in accordance with an important upper bound,

$\left|\frac{\alpha(s)}{\sqrt{s} \ln s}\right| \leq$ const,$\quad s \rightarrow \infty$.

In our study of central production, the direct-channel pole decomposition of the dual amplitude $A\left(M_{X}^{2}, t\right)$ is relevant. Different trajectories $\alpha_{i}\left(M_{X}^{2}\right)$ contribute to this amplitude, with $\alpha_{i}\left(M_{X}^{2}\right)$ a nonlinear, complex Regge trajectory in the Pomeron-Pomeron system,

$A\left(M_{X}^{2}, t\right)=a \sum_{i=f, P} \sum_{J} \frac{\left[f_{i}(t)\right]^{J+2}}{J-\alpha_{i}\left(M_{X}^{2}\right)}$.

In Eq. (3), the pole decomposition of the dual amplitude $A\left(M_{X}^{2}, t\right)$ is shown with $t$ the squared momentum transfer in the $P P \rightarrow P P$ reaction. The index $i$ sums over the trajectories which contribute to the amplitude. Within each trajectory, the second sum extends over the bound states of spin $J$. The prefactor $a$ in Eq. (3) has the numerical value $a=$ $1 \mathrm{GeV}^{-2}=0.389 \mathrm{mb}$.

The pole residue $f(t)$ appearing in the $P P \rightarrow P P$ system is fixed by the dual model, in particular by the compatibility of its Regge asymptotics with Bjorken scaling and reads

$f(t)=\left(1-t / t_{0}\right)^{-2}$,

where $t_{0}$ is a parameter to be fitted to the data. However, due to the absence of data so far, we set $t_{0}=0.71 \mathrm{GeV}^{2}$ for the moment as in the proton elastic form factor. Note that the residue enters with a power $(J+2)$ in Eq. (3), thereby strongly damping higher spin resonance contributions. The imaginary part of the amplitude $A\left(M_{X}^{2}, t\right)$ given in Eq. (3) is defined by

$$
\begin{aligned}
& \Im m A\left(M_{X}^{2}, t\right) \\
& =a \sum_{i=f, P} \sum_{J} \frac{\left[f_{i}(t)\right]^{J+2} \Im m \alpha_{i}\left(M_{X}^{2}\right)}{\left(J-\operatorname{Re} \alpha_{i}\left(M_{X}^{2}\right)\right)^{2}+\left(\Im m \alpha_{i}\left(M_{X}^{2}\right)\right)^{2}} .
\end{aligned}
$$

For the $P P$ total cross section we use the norm

$\sigma_{t}^{P P}\left(M_{X}^{2}\right)=\Im m A\left(M_{X}^{2}, t=0\right)$,

and we recall that the amplitude $A$ and the cross section $\sigma_{t}$ carry dimensions of $\mathrm{mb}$ due to the dimensional parameter $a$ discussed above. The Pomeron-Pomeron channel, $P P \rightarrow$ $M_{X}^{2}$, couples to the Pomeron and $f$ channels dictated by conservation of the quantum numbers. For calculating the $P P$ cross section, we hence take into account the trajectories associated to the $f_{0}(980)$ and the $f_{2}(1270)$ resonance, and the Pomeron trajectory.

\section{Nonlinear, complex meson Regge trajectories}

A non-trivial task for analytic models of Regge trajectories consists in deriving the imaginary part of the trajectory from the seemingly linearly increasing real part [24-26]. The importance of the nonlinearity of the real part was studied in Refs. [27-29]. A dispersion relation connects the real and imaginary part of the trajectory.

We follow Ref. [25] to relate the nearly linear real part of the meson trajectory to its imaginary part,

$\Re e \alpha(s)=\alpha(0)+\frac{s}{\pi} P V \int_{0}^{\infty} \mathrm{d} s^{\prime} \frac{\Im m \alpha\left(s^{\prime}\right)}{s^{\prime}\left(s^{\prime}-s\right)}$.

In Eq. (7), PV denotes the Cauchy Principal Value of the integral. The imaginary part is related to the decay width by 
$\Gamma\left(M_{R}\right)=\frac{\Im m \alpha\left(M_{R}^{2}\right)}{\alpha^{\prime} M_{R}}$.

The quantity $\alpha^{\prime}$ in Eq. (8) denotes the derivative of the real part, $\alpha^{\prime}=\frac{\mathrm{d} \Re e \alpha(s)}{\mathrm{d} s}$. The relation between $\Gamma(M)$ and $\Im m \alpha(\mathrm{s})$ requires $\Im m \alpha(s)>0$. In a simple analytical model, the imaginary part is chosen as a sum of single threshold terms [25]

$\Im m \alpha(s)=\sum_{n} c_{n}\left(s-s_{n}\right)^{1 / 2}\left(\frac{s-s_{n}}{s}\right)^{\left|\Re e \alpha\left(s_{n}\right)\right|} \theta\left(s-s_{n}\right)$.

The imaginary part of the trajectory displayed in Eq. (9) has the correct threshold and asymptotic behaviour. Since $\Im m \alpha(s)>0$, all the expansion coefficients $c_{\mathrm{n}}$ must be positive. The values of $s_{\mathrm{n}}$ represent kinematical thresholds of decay channels. The highest threshold, higher than all the resonance masses lying on the trajectory, is chosen as an effective threshold. This highest threshold ensures that $\Re e \alpha(\mathrm{s})$ tends to a constant value for $s \rightarrow \infty$.

The parameterisation of the real and imaginary part of a meson trajectory, and the extraction of the expansion coefficients $\mathrm{c}_{n}$ shown in Eq. (9), are derived in Appendix A for the case of the $\rho$-a trajectory.

\section{Two $f$ trajectories}

Apart from the Pomeron trajectory discussed below, the direct-channel $f$ trajectory is essential in the PP system. Guided by conservation of quantum numbers, we include two $f$ trajectories, labelled $f_{1}$ and $f_{2}$, with mesons lying on these trajectories as specified in Table 1 .

The real and imaginary part of the $f_{1}$ and $f_{2}$ trajectories can be derived as discussed in Appendix A from the parameters of the f-resonances in Table 1.

The real part and the width function of the $f_{1}$ trajectory are shown in Fig. 6 at the top and at the bottom, respectively. In order to fit this trajectory, the same three thresholds are used as for the $\rho$-a trajectory (see Appendix A).

The real part and the width function of the $f_{2}$ trajectory are shown in Fig. 7 at the top and bottom, respectively. In the

Table 1 Parameters of resonances belonging to the $f_{1}$ and $f_{2}$ trajectories

\begin{tabular}{lllll}
\hline & $\mathrm{I}^{G} \mathrm{~J}^{P C}$ & Traj. & $\mathrm{M}(\mathrm{GeV})$ & $\Gamma(\mathrm{GeV})$ \\
\hline$f_{0}(980)$ & $0^{+} 0^{++}$ & $f_{1}$ & $0.990 \pm 0.020$ & $0.070 \pm 0.030$ \\
$f_{1}(1420)$ & $0^{+} 1^{++}$ & $f_{1}$ & $1.426 \pm 0.001$ & $0.055 \pm 0.003$ \\
$f_{2}(1810)$ & $0^{+} 2^{++}$ & $f_{1}$ & $1.815 \pm 0.012$ & $0.197 \pm 0.022$ \\
$f_{4}(2300)$ & $0^{+} 4^{++}$ & $f_{1}$ & $2.320 \pm 0.060$ & $0.250 \pm 0.080$ \\
$f_{2}(1270)$ & $0^{+} 2^{++}$ & $f_{2}$ & $1.275 \pm 0.001$ & $0.185 \pm 0.003$ \\
$f_{4}(2050)$ & $0^{+} 4^{++}$ & $f_{2}$ & $2.018 \pm 0.011$ & $0.237 \pm 0.018$ \\
$f_{6}(2510)$ & $0^{+} 6^{++}$ & $f_{2}$ & $2.469 \pm 0.029$ & $0.283 \pm 0.040$ \\
\hline
\end{tabular}
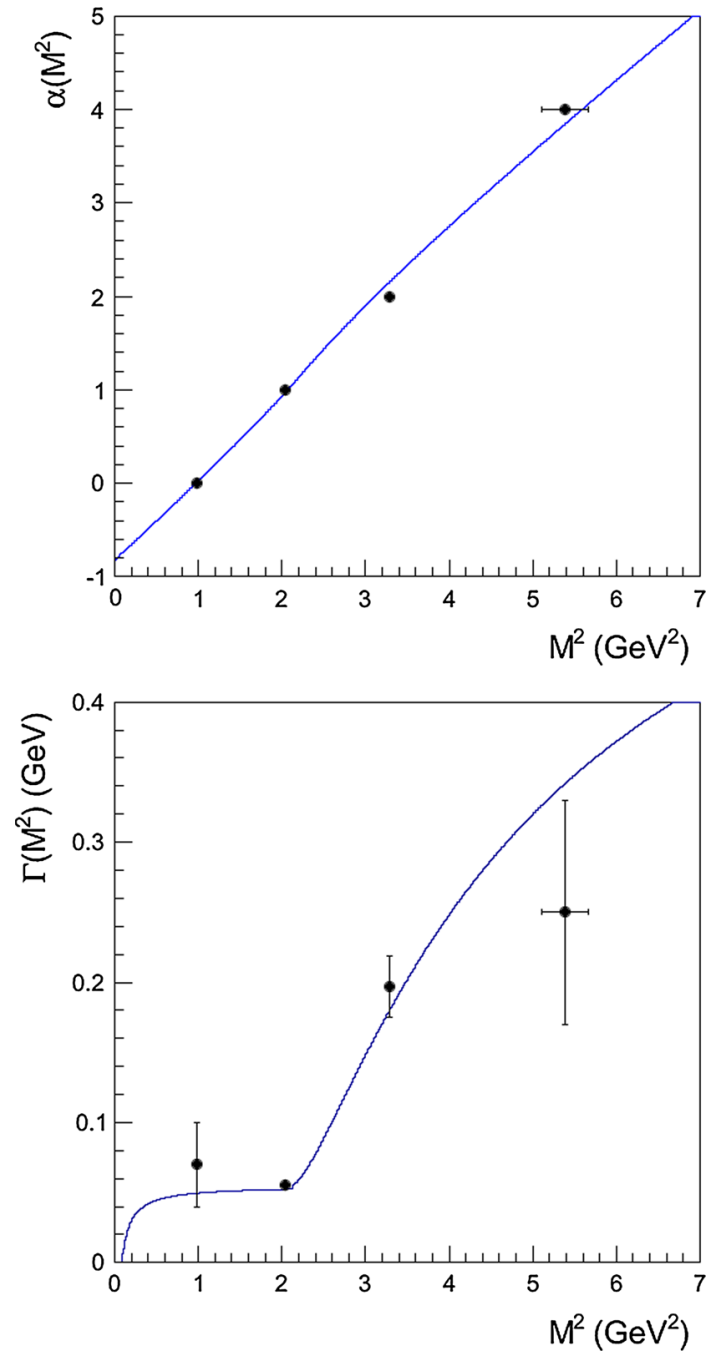

Fig. 6 Real part of $f_{1}$ trajectory at the top, width function $\Gamma\left(\mathrm{M}^{2}\right)$ at the bottom

fit of this trajectory $f_{2}$, the same three thresholds are used as for the $f_{1}$ trajectory.

\section{The Pomeron trajectory}

While ordinary meson trajectories can be fitted both in the resonance and scattering region corresponding to positive and negative values of the argument, the parameters of the Pomeron trajectory can only be determined in the scattering region $M^{2}<0$. The poles of this trajectory at $M^{2}>0$ are identified with glueball candidates. An extensive literature on such candidates exists, including theoretical predictions and experimental identification. The status of glueballs is, however, controversial and a topic of ongoing discussions and debate; see Refs. $[7,30]$ and the references therein. Here, we associate the bound states of the Pomeron trajectory to glueball candidates, as previously done in Refs. [29,31-33]. 

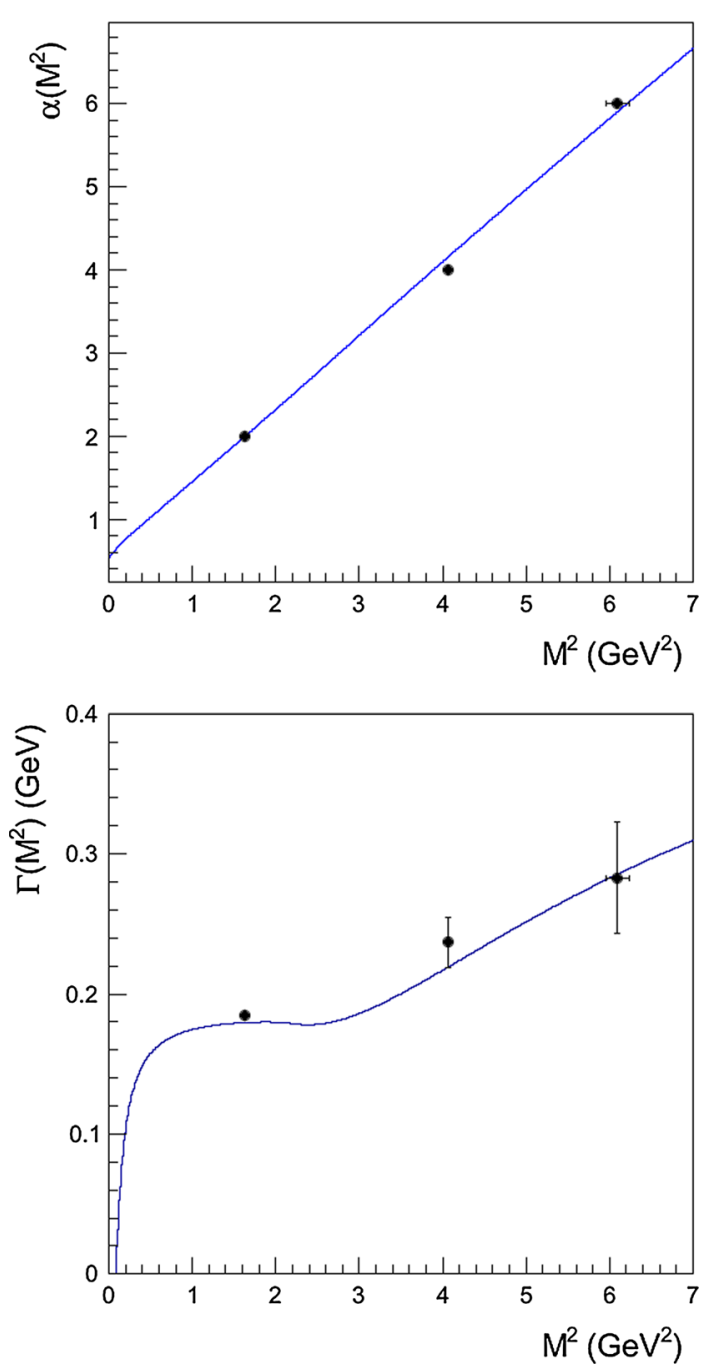

Fig. 7 Real part of $f_{2}$ trajectory at the top, width function $\Gamma\left(\mathrm{M}^{2}\right)$ at the bottom

A comprehensive fit to high-energy $p p$ and $p \bar{p}$ of the nonlinear Pomeron trajectory is discussed in Ref. [14]

$\alpha_{P}\left(M^{2}\right)=1 .+\varepsilon+\alpha^{\prime} M^{2}-c \sqrt{s_{0}-M^{2}}$,

with $\varepsilon=0.08, \alpha^{\prime}=0.25 \mathrm{GeV}^{-2}$ and $s_{0}$ the two-pion threshold $s_{0}=4 m_{\pi}^{2}$. The value of $c$ is $c=\alpha^{\prime} / 10=0.025$.

In order to be consistent with the mesonic trajectories shown above, the linear term in Eq. (10) is replaced by a heavy threshold mimicking linear behaviour in the mass region of interest $(M<5 \mathrm{GeV})$,

$$
\begin{aligned}
\alpha_{P}\left(M^{2}\right)= & \alpha_{0}+\alpha_{1}\left(2 m_{\pi}-\sqrt{4 m_{\pi}^{2}-M^{2}}\right) \\
& +\alpha_{2}\left(\sqrt{M_{\mathrm{H}}^{2}}-\sqrt{M_{\mathrm{H}}^{2}-M^{2}}\right),
\end{aligned}
$$

where $M_{\mathrm{H}}$ is an effective heavy threshold set at $M=3.5 \mathrm{GeV}$. The coefficients $\alpha_{0}, \alpha_{1}$ and $\alpha_{2}$ are chosen such that the
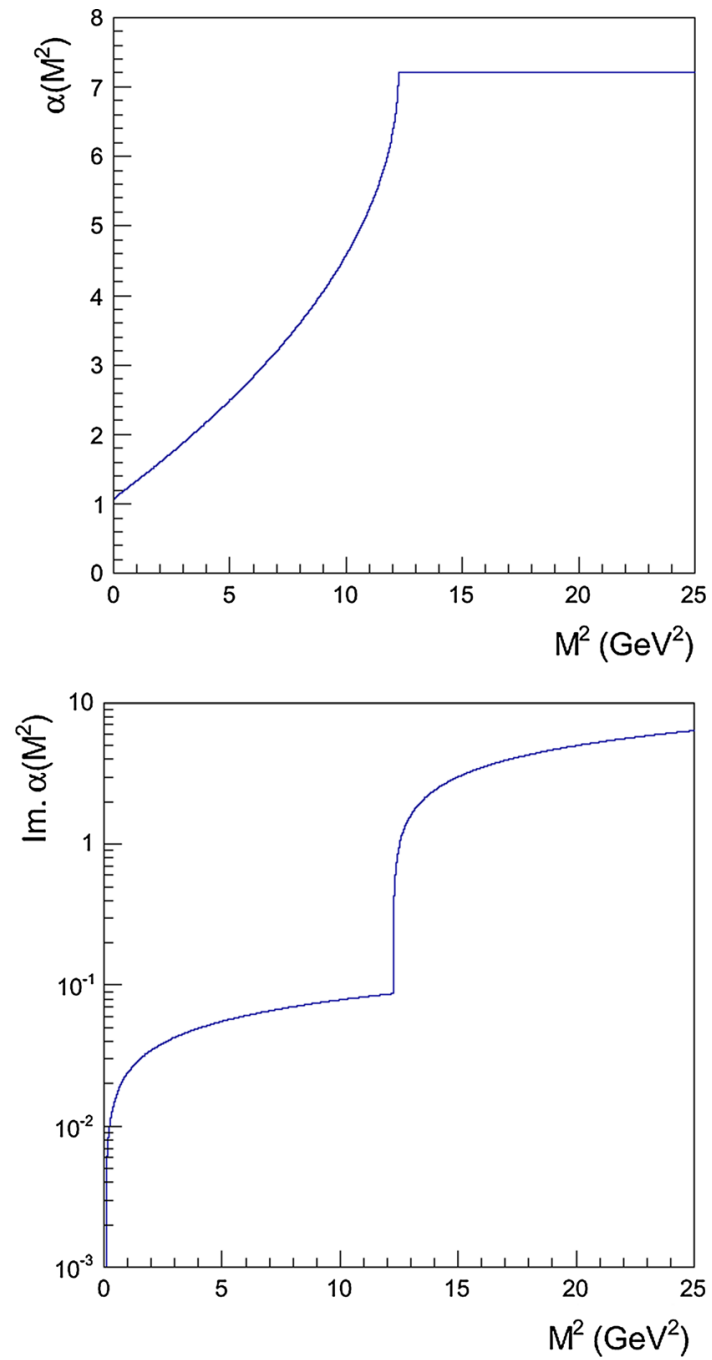

Fig. 8 Real part of Pomeron trajectory at the top, imaginary part at the bottom

Pomeron trajectory of Eq. (11) has a low energy behaviour as defined by Eq. (10).

The real and imaginary part of the Pomeron trajectory resulting from the parameterisation of Eq. (11) are shown in Fig. 8 at the top and bottom, respectively. Clearly visible is the asymptotically constant value of the real part beyond the heavy threshold, accompanied by a strong increase of the imaginary part.

\section{The $f_{0}(500)$ resonance}

The experimental data on central exclusive pion-pair production measured at the energies of the ISR, RHIC, TEVATRON and the LHC collider all show a broad continuum for pair masses $\mathrm{m}_{\pi^{+} \pi^{-}}<1 \mathrm{GeV}$. This mass region is experimentally difficult to access due to the missing acceptance for pairs of low mass and low transverse momentum $\mathrm{p}_{T}$ discussed above. The population of this mass region is attributed to 
the $f_{0}(500)$, a resonance which has been controversial for many decades. In the 2010 edition of the Review of Particle Physics (RPP), this resonance is listed as $f_{0}(600)$ with a mass $M_{0}$ in the range $400<M_{0}<1200 \mathrm{MeV}$, and a width $\Gamma$ in the range $600<\Gamma<1000 \mathrm{MeV}$. Since the RPP edition of 2012, this resonance is listed as $f_{0}(500)$ with mass in the range $400<M_{0}<550 \mathrm{MeV}$, and width in the range $400<\Gamma<700 \mathrm{MeV}$ [34].

The $f_{0}(500)$ resonance is of prime importance for the understanding of the attractive part of the nucleon-nucleon interaction, as well as for the mechanism of spontaneous breaking of chiral symmetry. The nature of the $f_{0}(500)$ is a topic of ongoing studies and discussions, it is, however, generally agreed that it cannot be interpreted as a predominant $q \bar{q}$-state. The non-ordinary nature of the $f_{0}(500)$ resonance is corroborated by the fact that it does not fit into the Regge description of classifying $q \bar{q}$-states into trajectories [35]. A possible interpretation of the $f_{0}(500)$ is a tetra-quark configuration consisting of two valence and two antiquarks in the colour-neutral state. It was shown that such a configuration can give rise to a nonet of light scalar-isoscalar mesons [36]. Different approaches interpret the $f_{0}(500)$ as arising from an inner tetra-quark structure and changing to an outer structure of a pion-pion state [37]. There is strong evidence that this $f_{0}(500)$ state belongs to a $\mathrm{SU}(3)$ nonet composed of the $f_{0}(500), f_{0}(980), a_{0}(980)$ and $K_{0}^{*}(800)$.

In spite of the complexity of the $f_{0}(500)$ resonance, and the controversy on its interpretation and description, we take here the practical but simple-minded approach of a BreitWigner resonance [38],

$A\left(M^{2}\right)=a \frac{-M_{0} \Gamma}{M^{2}-M_{0}^{2}+i M_{0} \Gamma}$.

In Eq. (12), the parameterisation of the relativistic BreitWigner amplitude is shown with $M_{0}$ and $\Gamma$ the mass and width, respectively. Here, the prefactor $a$ is added for consistency with the definition of the amplitude shown in Eq. (3). The Breit-Wigner amplitude of Eq. (12) is used below for calculating the contribution of the $f_{0}(500)$ resonance to the PP cross section.

\section{Pomeron-Pomeron total cross section}

The PP cross section is calculated from the imaginary part of the amplitude by use of the optical theorem,

$$
\begin{aligned}
& \sigma_{t}^{P P}\left(M^{2}\right) \\
& =\Im m A\left(M^{2}, t=0\right) \\
& \quad=a \sum_{i=f, P} \sum_{J} \frac{\left[f_{i}(0)\right]^{J+2} \Im m \alpha_{i}\left(M^{2}\right)}{\left(J-\Re e \alpha_{i}\left(M^{2}\right)\right)^{2}+\left(\Im m \alpha_{i}\left(M^{2}\right)\right)^{2}} .
\end{aligned}
$$

In Eq. (13), the index $i$ sums over the trajectories which contribute to the cross section, in our case the $f_{1}, f_{2}$ and the Pomeron trajectory. Within each trajectory, the summation extends over the poles of spin $J$ as expressed by the second summation sign. The value $f_{i}(0)=\left.f_{i}(t)\right|_{t=0}$ is not known a priori, but can, however, be extracted from the experimental data by analysing relative strengths of resonances within a trajectory.

The Breit-Wigner parameterisation of the isolated $f_{0}(500)$ resonance contributes to the cross section with

$\sigma_{f_{0}(500)}^{P P}\left(M^{2}\right)=a \sqrt{1 .-\frac{4 m_{\pi}^{2}}{M^{2}}} \frac{M_{0}^{2} \Gamma^{2}}{\left(M^{2}-M_{0}^{2}\right)^{2}+M_{0}^{2} \Gamma^{2}}$,

with the resonance mass of $M_{0}=(0.40-0.55) \mathrm{GeV}$ and a width $\Gamma=(0.40-0.70) \mathrm{GeV}$ [38]. The quantity $\sqrt{1 .-4 m_{\pi}^{2} / M^{2}}$ in Eq. (14) is the threshold phase space factor for the two-pion decay.

In addition to the contributions discussed above, a background term is added to the PP cross section. This background is of form [39]

$\sigma_{\text {backgr. }}^{P P}\left(M^{2}\right)=c *\left(0.1+\log \left(M^{2}\right)\right) \mathrm{mb}$,

with the numerical value of the parameter $c$ fitted to data.

In Fig. 9, the different contributions to the PP total cross section are shown. The contribution of the $f_{0}(500)$ resonance according to Eq. (14) is displayed by the dashed cyan line. Here, the central values are taken for the mass $M_{0}$ as well as for the width $\Gamma, M_{0}=475 \mathrm{MeV}$ and $\Gamma=550 \mathrm{MeV}$, respectively. The contribution of the $f_{1}$ trajectory indicated by the solid green line clearly shows the $f_{0}(980)$ and the $f_{1}(1420)$ resonance. The higher mass states, the $f_{2}(1810)$ and the $f_{4}(2300)$, are barely visible due to their reduced cross section and much larger width. Similarly, the contribution of the $f_{2}$ trajectory indicated by the dashed blue line shows peaks for the $f_{2}(1270)$ and the $f_{4}(2050)$ resonances, with the $f_{6}(2510)$ barely visible. The contributions of both $f_{1}$ and $f_{2}$ trajectory show a kink at about $M=5.5 \mathrm{GeV}$ due to the heavy threshold $s_{2}=30 \mathrm{GeV}^{2}$. The contribution from the Pomeron trajectory is displayed in Fig. 9 by the dashed magenta line. Visible is the resonance structure due to the $J=2,4$ and 6 states on the trajectory labelled by $\operatorname{gb}(J=2), \operatorname{gb}(J=4)$ and $\operatorname{gb}(J=6)$, respectively. Beyond the heavy threshold, $M=3.5 \mathrm{GeV}$, the transition to the continuum is seen, reflecting the behaviour of the real and imaginary part of the trajectory as shown in Fig. 8. The background contribution to the PP cross section is shown in Fig. 9 by the dashed black line, and is normalized here to represent approximately $10 \%$ of the signal at $M=7 \mathrm{GeV}$.

The Pomeron-Pomeron total cross section is calculated by summing over the contributions discussed above, and is shown in Fig. 9 by the solid black line. The prominent structures seen in the total cross section are labelled by the reso- 
Fig. 9 Contributions of the $f_{0}(500)$ resonance, the $f_{1}, f_{2}$ and the Pomeron trajectory, and of the background to PP total cross section

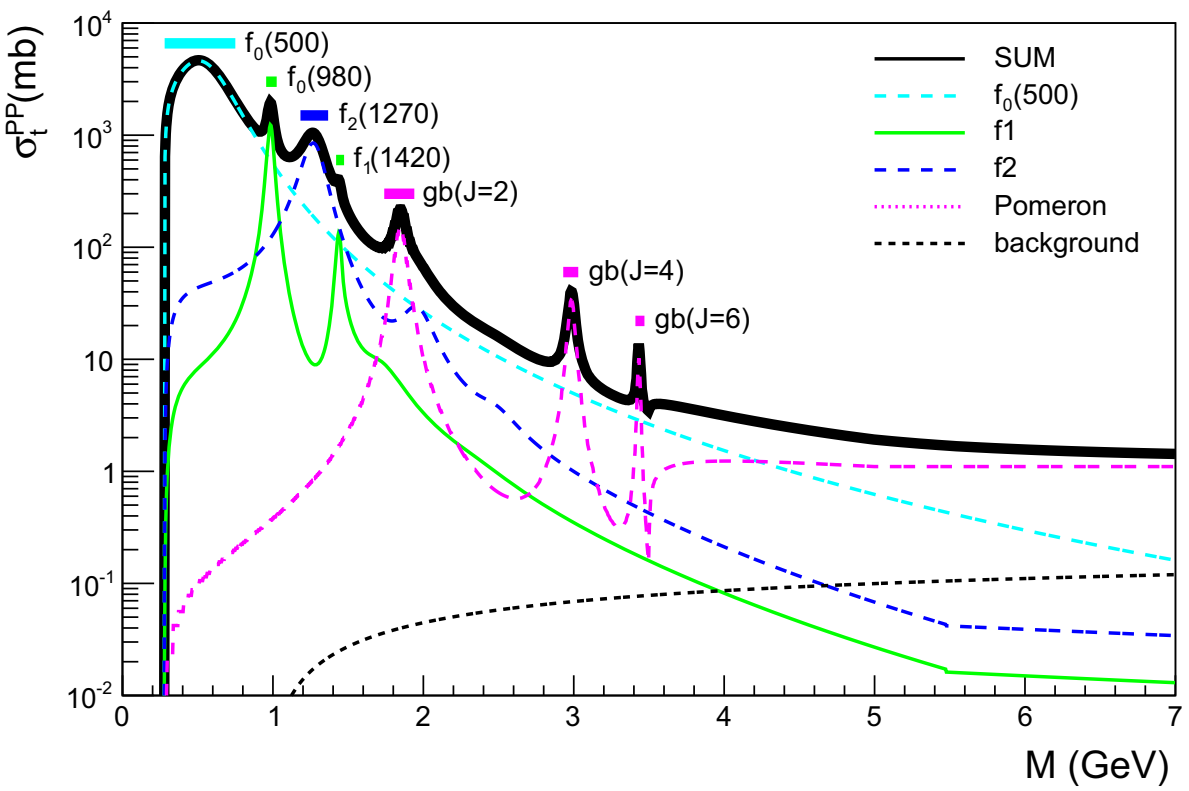

nances generating the peaks. The model presented here does not specify the relative strength of the different contributions shown in Fig. 9. A Partial Wave Analysis of experimental data on central production events will be able to extract the quantum numbers of these resonances, and it will hence allow one to associate each resonance to its trajectory. The relative strengths of the contributing trajectories need to be determined from the experimental data.

\section{Summary and outlook}

A Regge pole model is presented for calculating the Pomeron-Pomeron total cross section in the resonance region $\sqrt{M^{2}} \leq 5 \mathrm{GeV}$. The direct-channel contributions of the Pomeron and two $f$ trajectories, including a background, are presented. The resonance region $\sqrt{M^{2}} \leq 1 \mathrm{GeV}$ is described by a Breit-Wigner parameterisation of the $f_{0}(500)$ resonance. The relative strength of these contributions cannot be specified within the model, and must hence be determined from the analysis of experimental data. The model presented allows an extension to central production of strangeonia and charmonia states by taking into account the direct-channel contribution of the respective trajectories. Moreover, this model can be extended to lower beam energies where not only Pomeron-Pomeron, but also PomeronReggeon and Reggeon-Reggeon diagrams need to be considered. The result of the presented work is only the starting point for a comprehensive study of central exclusive production. To make measurable predictions for the LHC, all the diagrams shown in Fig. 3 must be calculated. The results presented here are necessary and essential input for such calculations. Anticipating further studies, we recall of the possible reference points that can be used as a guide. For the absolute value we use the asymptotic value $\sigma_{t} \approx 1 \mathrm{mb}$, compatible with both QCD-inspired and phenomenological estimates [40,41]. The Pomeron-Pomeron total cross section depends also on Pomerons' virtualities, $t_{1}, t_{2}$. We ignored this dependence for two reasons: First, this dependence is known at best at their high values, where perturbative QCD results, such as that of Eq. (1), may be valid. Second, for simplicity, we fix this dependence, including it as part of the normalisation factor. Varying the $t$ dependence and the partition between $t_{1}$ and $t_{2}$ may be attempted to account for by, following Eq. (1), simply dividing Eq. (3) by $\sqrt{t_{1} t_{2}}$, this may be true only for high values of $t_{i}$, beyond diffraction.

Acknowledgments We thank Risto Orava and Alessandro Papa for discussions. This work is supported by the German Federal Ministry of Education and Research under promotional reference 05P15VHCA1. One of us (L. J.) gratefully acknowledges an EMMI visiting Professorship at the University of Heidelberg for completion of this work.

Open Access This article is distributed under the terms of the Creative Commons Attribution 4.0 International License (http://creativecomm ons.org/licenses/by/4.0/), which permits unrestricted use, distribution, and reproduction in any medium, provided you give appropriate credit to the original author(s) and the source, provide a link to the Creative Commons license, and indicate if changes were made. Funded by $\mathrm{SCOAP}^{3}$.

\section{Appendix A}

Although the $\rho$-a trajectory does not couple to the $P P$ channel, and hence does not contribute to the $P P$ total cross section, we list it here to present the procedure for extracting the coefficients $\mathrm{c}_{n}$ in the expansion of the trajectory. For convenience, we repeat here the Ansatz for the imaginary part of the trajectory expressed in Eq. (9) 
$\Im m \alpha(s)=\sum_{n} c_{n}\left(s-s_{n}\right)^{1 / 2}\left(\frac{s-s_{n}}{s}\right)^{\left|\Re e \alpha\left(s_{n}\right)\right|} \theta\left(s-s_{n}\right)$.

The real part $\Re e \alpha(s)$ of the trajectory expressed by Eq. (7) can be calculated as

$$
\begin{aligned}
\Re e \alpha(s)= & \alpha(0)+\frac{s}{\sqrt{\pi}} \sum_{n} c_{n} \frac{\Gamma\left(\lambda_{n}+3 / 2\right)}{\Gamma\left(\lambda_{n}+2\right) \sqrt{s_{n}}} \\
& \times{ }_{2} F_{1}\left(1,1 / 2 ; \lambda_{n}+2 ; \frac{s}{s_{n}}\right) \theta\left(s_{n}-s\right) \\
& +\frac{2}{\sqrt{\pi}} \sum_{n} c_{n} \frac{\Gamma\left(\lambda_{n}+3 / 2\right)}{\Gamma\left(\lambda_{n}+1\right)} \sqrt{s_{n}} \\
& \times{ }_{2} F_{1}\left(-\lambda_{n}, 1 ; 3 / 2 ; \frac{s_{n}}{s}\right) \theta\left(s-s_{n}\right) .
\end{aligned}
$$

The derivative $\Re e \alpha^{\prime}$ (s) can be derived from Eq. (17)

$$
\begin{aligned}
\Re e \alpha^{\prime}(s)= & \frac{1}{\sqrt{\pi}} \sum_{n} c_{n} \frac{\Gamma\left(\lambda_{n}+3 / 2\right)}{\Gamma\left(\lambda_{n}+2\right) \sqrt{s_{n}}} \\
& \times{ }_{2} F_{1}\left(2,1 / 2 ; \lambda_{n}+2 ; \frac{s}{s_{n}}\right) \theta\left(s_{n}-s\right) \\
& +\frac{4}{3 \sqrt{\pi}} \sum_{n} c_{n} \frac{\Gamma\left(\lambda_{n}+3 / 2\right)}{\Gamma\left(\lambda_{n}\right)} \frac{s_{n}^{3 / 2}}{s^{2}} \\
& \times{ }_{2} F_{1}\left(1-\lambda_{n}, 2 ; 5 / 2 ; \frac{s_{n}}{s}\right) \theta\left(s-s_{n}\right) .
\end{aligned}
$$

Equations (16)-(18) are used to calculate the parameters $c_{n}$ of $\Re e \alpha$, Re $\alpha^{\prime}$ and $\Im m \alpha$ by a $\chi^{2}$-fit procedure. A linear fit to the real part provides start values for $\alpha(0)$ and $\Re e \alpha^{\prime}$, from which start values for $\Im m \alpha\left(M_{R}^{2}\right)$ are calculated.

The fit of the parameters $c_{n}$ is done in three steps. First, the $\mathrm{c}_{\mathrm{n}}$ are fitted to the expression of the imaginary part $\Im m \alpha(\mathrm{s})$ as given in Eq. (16). Second, the coefficients $\alpha(0)$ and $c_{2}$ are extracted by using the parameterisation of the real part $\Re e \alpha(\mathrm{s})$ as defined by Eq. (17). Third, new values are calculated for $\Re e \alpha\left(s_{n}\right)$, and the imaginary part $\Im m \alpha(\mathrm{s})$ is updated according to Eq. (16). These three steps can be repeated if necessary until convergence of the values $\alpha(0)$ and $c_{n}$ is reached.

For fitting the $\rho$-a trajectory, we take the same three thresholds $s_{\mathrm{i}}$ as outlined in Ref. [25]. The lowest value $s_{0}$ is taken

Table 2 Parameters of $\rho$ - and a-resonances

\begin{tabular}{llll}
\hline & $I^{G} J^{P C}$ & $M(\mathrm{GeV})$ & $\Gamma(\mathrm{GeV})$ \\
\hline$\rho(770)$ & $1^{+} 1^{--}$ & $0.769 \pm 0.001$ & $0.149 \pm 0.001$ \\
$\rho_{3}(1690)$ & $1^{+} 3^{--}$ & $1.688 \pm 0.002$ & $0.161 \pm 0.010$ \\
$\rho_{5}(2350)$ & $1^{+} 5^{--}$ & $2.330 \pm 0.035$ & $0.400 \pm 0.100$ \\
$a_{2}(1320)$ & $1^{-} 2^{++}$ & $1.319 \pm 0.001$ & $0.105 \pm 0.002$ \\
$a_{4}(2040)$ & $1^{-} 4^{++}$ & $1.996 \pm 0.010$ & $0.255 \pm 0.026$ \\
$a_{6}(2450)$ & $1^{-} 6^{++}$ & $2.450 \pm 0.130$ & $0.400 \pm 0.250$ \\
\hline
\end{tabular}
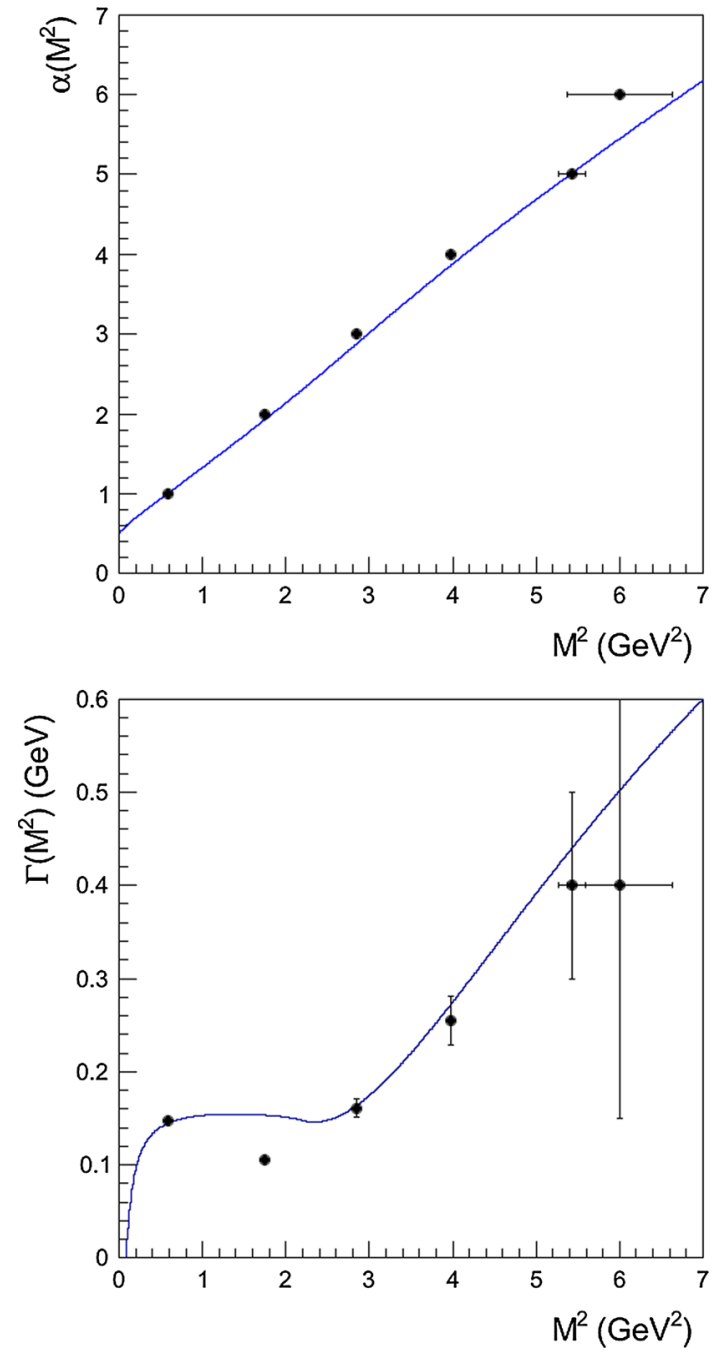

Fig. 10 Real part of $\rho$-a trajectory at the top, width function $\Gamma\left(\mathrm{M}^{2}\right)$ at the bottom

as the 2-pion threshold $s_{0}=4 \mathrm{~m}_{\pi}^{2}$, with the second value $s_{1}$ defined by the a 2 (1320)- $\pi$ threshold, $s_{1}=2.12 \mathrm{GeV}^{2}$. The highest threshold $s_{2}$ is taken as $s_{2}=30 \mathrm{GeV}^{2}$.

The parameters of the resonances used for the fit of the $\rho$-a trajectory are shown in Table 2 . In Fig. 10, the resulting real part and the width function $\Gamma\left(M^{2}\right)$ are shown.

At the top of Fig. 10, the black dots represent a plot of the squared masses $M^{2}$ of the resonances $\rho_{0}(770), \rho_{3}(1670)$, $\rho_{5}$ (2350), $a_{2}$ (1320), $a_{4}(2040)$ and $a_{6}(2450)$ versus their spin. The seemingly linear correlation between these two variables, $\alpha\left(M^{2}\right)=\alpha_{0}+\alpha^{\prime}\left(M^{2}\right)$, is clearly shown by the solid curve as determined from Eq. (17). For comparison, the star symbols superimposed in Fig. 10 at the top represent the states $\omega_{1}(782), \omega_{3}(1670), f_{2}(1270), f_{4}(2050)$ and $f_{6}(2510)$. The top part of Fig. 10 clearly illustrates the approximate degeneracy of the $\rho$-, $\omega$-, $f$ - and $a$-trajectory. The width function $\Gamma\left(\mathrm{M}^{2}\right)$ of the $\rho$-a trajectory shown in Fig. 10 at the bottom shows good agreement with the corresponding width function of Ref. [25]. 


\section{References}

1. A. Austregesilo, for the COMPASS Collaboration. Proceedings 15 th conference on elastic and diffractive scattering, Saariselka, Sept 2013, arXiv: 1310.3190

2. M. Albrow, For the CDF collaboration, Int. J. Mod. Phys. A29(28), 1446009 (2014). arXiv: 1409.0462

3. L. Adamczyk, W. Guryn, J. Turnau, Int. J. Mod. Phys. A29(28), 1446010 (2014). arXiv: 1410.5752

4. R. Schicker, Int. J. Mod. Phys. A 29(28), 1446015 (2014). arXiv: 1411.1283

5. R. McNulty, Int. J. Mod. Phys. A 29(28), 1446003 (2014). arXiv: 1409.8113

6. M. Albrow, V. Khoze, Ch. Royon, Spec. Issue Int. J. Mod. Phys. A 29, 28 (2014)

7. W. Ochs, J. Phys. G40, 043001 (2013). arXiv:1301.5183 [hep-ph]

8. M. Zurek, for the CDF Collaboration, WE-Heraeus Physics School, Bad Honnef, 17-21 August 2015. http://school-diff2015.physi. uni-heidelberg.de/Talks/Zurek.pdf

9. A. Austregesilo, for the COMPASS Collaboration. PoS Hadron. 2013, 102 (2013). arXiv:1402.2170

10. CDF Collaboration, Phys. Rev. D 91(9), 091101 (2015). arXiv: 1502.01391

11. R. Schicker, for the ALICE Collaboratiom, 14th International Conference on Hadron Spectroscopy: Hadron 2011, 13-17 June 2011, Munich, Germany. arXiv:1110.3693

12. L. Jenkovszky, O. Kuprash, J.W. Lamsa, V.K. Magas, R. Orava, Phys. Rev. D 83, 056014 (2011). arXiv: 1011.0664

13. L. Jenkovszky et al. Yad. Fizika, 77, 1535 (2014). arXiv:1211.5841

14. L. Jenkovszky, Rivista Nuovo Cim. 10, N12 (1987)

15. A. Donnachie, P. Landshoff, Phys. Lett. B 750, 669 (2015). arXiv: 1309.1292

16. C. Ewerz, M. Maniatis, O. Nachtmann, Ann. Phys. 342, 31 (2014). arXiv: 1309.3478

17. P. Lebiedowicz, O. Nachtmann, A. Szczurek, Ann. Phys. 344, 301 (2014). arXiv:1309.3913

18. A. Bolz, C. Ewerz, M. Maniatis, O. Nachtmann, M. Sauter, A. Schöning, JHEP 1501, 151 (2015). arXiv: 1409.8483

19. J. Bartels, A. De Roeck, H. Lotter, Phys. Lett. B389, 742 (1996)

20. S.J. Brodsky, V.S. Fadin, V.T. Kim, L.N. Lipatov, G.B. Pivovarov, J. Exp. Theor. Phys. Lett. 76, 249 (2002)
21. F. Caporale, D. Y. Ivanov, A. Papa, Eur. Phys. J. C 58, 1 (2008)

22. R. Fiore, A. Flachi, L.L. Jenkovszky, A. Lengyel, V. Magas, Phys. Rev. D 69, 014004 (2004). arXiv:hep-ph/0308178

23. A.I. Bugrij et al., Fortschr. Phys. 21, 427 (1973)

24. A. Degasperis, E. Predazzi, Nuovo Cim. Vol. A 65, 764-782 (1970)

25. R. Fiore, L.L. Jenkovszky, V. Magas, F. Paccanoni, A. Papa, Eur. Phys. J. A 10, 217-221 (2001). arXiv:hep-ph/0011035

26. R. Fiore, L.L. Jenkovszky, F. Paccanoni, A. Prokudin, Phys. Rev. D 70, 054003 (2004). arXiv:hep-ph/0404021

27. S. Filipponi, G. Pancheri, Y. Srivastava, Phys. Rev. D 59, 076003 (1999). arXiv:hep-ph/9804270

28. R. Fiore, L.L. Jenkovszky, V. Magas, F. Paccanoni, A. Papa, Phys. Part. Nucl. 31, 46 (2000). arXiv:hep-ph/9911503

29. M.M. Brisudova, L. Burakovsky, J.T. Goldman, Phys. Rev. D 61, 054013 (2000). arXiv:hep-ph/9906293

30. A. Kirk, Int. J. Mod. Phys. A 29(28), 1446001 (2014). arXiv: 1408.1196

31. A.B. Kaidalov, arXiv:hep-ph/9912434;

32. P. Desgrolard, L.L. Jenkvoszky, A.I. Lengyel, Where are the glueballs? ("Hadrons-94", Uzhgorod-Kiev, 1994), pp. 109-119

33. Sergienko, arXiv:1206.7091 [hep-ph]

34. J.R. Pelaez, arXiv: 1510.00653

35. A.V. Anisovich, V.V. Anisovich, A.V. Sarantsev, Phys. Rev. D 62, 051502 (2000). arXiv:hep-ph/0003113

36. R.L. Jaffe, Phys. Rev. D 15, 267 (1977)

37. F.E. Close, N.A. Tornqvist, J.Phys.G 28, (2002) R249, arXiv:hep-ph/0204205

38. K.A. Olive et al., (Particle Data Group), Chin. Phys. C 38, 090001 (2014) [2015 update]

39. L.L. Jenkovszky, S.Y. Kononenko, V.K Magas, Low-energy diffraction: a direct-channel point of view: the background. In "Diffraction 2002". Edited by R. Fiore et al., Kluwer Acadamic Publishers 2003

40. F.O. Duãres, N.S. Navarra, G. Wilk, Extracting the PomeronPomeron cross section from diffractive mass spectra. arXiv:hep-ph/0209149

41. R. Ciesielski, K. Goulianos, MBR Monte Carlo Simulation in PYTHIAS8. arXiv:1205.1446 [hep-ph] 